\title{
Extended Cerebral Infarction Due to Preoperative Free-Floating Thrombus in Right Internal Carotid Artery Complicated with Acute Type-A Aortic Dissection
}

\author{
Satoshi Yamashiro*, Ryoko Arakaki, Yuya Kise, Hitoshi Inafuku and Yukio Kuniyoshi \\ Department of Thoracic and Cardiovascular Surgery, Ryukyu University Hospital, Okinawa, Japan
}

\begin{abstract}
A 59-year-old man, who has cutaneous polyarteritis nodosa, presented with sudden onset of excruciating neck pain and syncope. Chest CT disclosed a Stanford type a acute aortic dissection. He rapidly lost consciousness and cardiac tamponade caused a drop in blood pressure. Emergency ascending aortic replacement proceeded under deep hypothermic circulatory arrest with antegrade selective cerebral perfusion and the cerebral blood supply was monitored throughout the procedure. However, post-operative brain $\mathrm{CT}$ imaging revealed extensive right hemispheric brain infarction. A large thrombus was identified in the right internal carotid artery. Whether the mechanism of brain ischemia associated with the aortic dissection was hemodynamic ischemia or thromboembolism remained unclear. We considered that thrombectomy might be needed before selective cerebral perfusion.
\end{abstract}

Keywords: Type A acute aortic dissection; Carotid artery thrombus; Organ malperfusion; Hypercoagulability

\section{Introduction}

Free-floating thrombus in the internal carotid artery is rare, its incidence is unknown and its management remains controversial. It is usually diagnosed by angiography after a symptomatic ischemic cerebral event. Moreover, neurological disorders such as the initial manifestation of type A aortic dissection become more difficult to diagnose. We describe ischemic stroke caused by thromboembolism with free-floating thrombus in the internal carotid artery due to type A aortic dissection.

\section{Case Report}

An ambulance delivered a 59-year-old man who developed sudden excruciating neck pain and syncope to the Emergency Room (ER) at our hospital. He has been diagnosed cutaneous polyarteritis nodosa and treated with steroid and immunosuppressant (cyclosporine and cyclophosphamide) for 3 years for department of Dermatology at our hospital. His serum examination revealed positive anticardiolipin antibody and lupus anticoagulant antibody. The other antibodies include antiprothrombin antibody and anti-Phosphatidylserine antibody, were negative. The primary ER physician assessed the head and neck using Computed Tomography (CT) and confirmed dissection of the aortic arch involving neck vessels. Assessment of the chest to the abdomen by CT imaging disclosed a Stanford type A acute aortic dissection extending from the ascending aorta to the bilateral common iliac arteries (Figure 1A). The left common carotid artery was significantly narrowed due to compression by a thrombus of the pseudo lumen. Moreover, a thrombosed formation was found at the innominate artery (Figure 1B). He lapsed into unconsciousness during a second CT assessment and cardiac tamponade caused a decrease in blood pressure. He was transferred to our department for surgery. We considered that rapid restoration of flow into the true lumen and obliteration of the false lumen would be the optimal approach to treat cerebral ischemia. We rapidly opened the pericardium to release the cardiac tamponade. The discharge of a large amount of bloody pericardial effusion immediately improved the blood pressure. A cardiopulmonary bypass was established with arterial cannulation from

the right axillary and right femoral arteries, and venous cannulation from the right atrium. Ascending aortic replacement proceeded under deep hypothermic circulatory arrest with Antegrade Selective Cerebral Perfusion (ASCP). An intimal tear was identified in the ascending aorta. The Right Coronary Artery (RCA) was ligated at the orifice because the dissection had extended at the right coronary cusp to include the RCA. We revascularized the RCA using a saphenous vein

A)

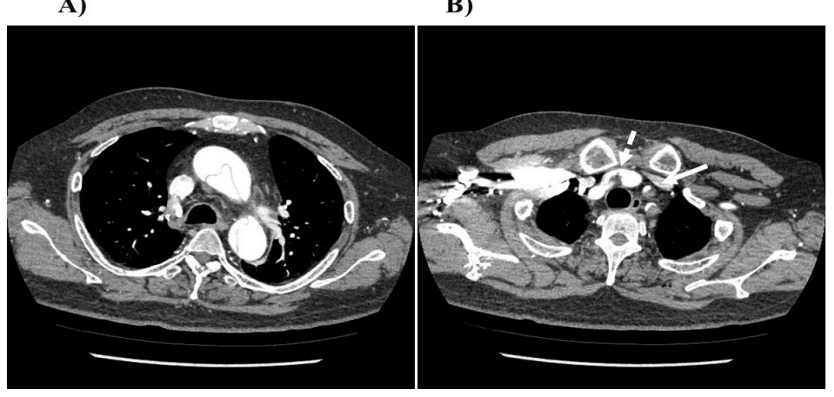

Figure 1: Preoperative Computed Tomography (CT) images.

A) Stanford type A acute aortic dissection extends from ascending aorta to bilateral common iliac arteries.

B) Left common carotid artery is significantly narrowed due to compression by thrombus in pseudo lumen (arrow). Thrombus is evident at innominate artery (dotted arrow).

${ }^{*}$ Corresponding author: Satoshi Yamashiro MD, PhD, Department of Thoracic and Cardiovascular Surgery, Ryukyu University Hospital, 207 Uehara, Nishihara, Nakagami, Okinawa 903-0215, Japan, Tel: (+81)-98-895-1168; Fax: (+81)-98-8951422; E-mail: y3104@med.u-ryukyu.ac.jp

Received December 19, 2013; Accepted January 23, 2014; Published January 25,2014

Citation: Yamashiro S, Arakaki R, Kise Y, Inafuku H, Kuniyoshi Y (2014) Extended Cerebral Infarction Due to Preoperative Free-Floating Thrombus in Right Internal Carotid Artery Complicated with Acute Type-A Aortic Dissection. J Vasc Med Surg 2: 126. doi: 10.4172/2329-6925.1000126

Copyright: (C) 2014 Yamashiro S, et al. This is an open-access article distributed under the terms of the Creative Commons Attribution License, which permits unrestricted use, distribution, and reproduction in any medium, provided the original author and source are credited. 
graft. Peri-operative blood flow through the middle cerebral arteries was continuously monitored using bilateral transcranial Doppler sonography (Viasys Inc., Conshocken, PA, USA). Moreover, cerebral oxygen saturation was monitored using an $\operatorname{Invos}^{\circledR}$ cerebral oximeter (Somanetics Corp., Troy, MI, USA). Both monitors ensured the supply of cerebral blood during the operation.

However, the patient did not awaken and convulsions occurred on Postoperative Day (POD) 2. Brain CT imaging revealed an extensive area of low-density in the right hemisphere (Figure 2A). The patient died of pneumonia on POD 90. A re-examination of the initial neck CT revealed a large free-floating thrombus in the right internal carotid artery that had existed before the operation (Figure 2B).

\section{Discussion}

Neurological manifestations of common carotid artery dissection, including transient ischemic attack and cerebral infarction, occur in $2.7 \%-7 \%$ of patients with aortic dissection [1]. Cerebral infarction could be due to common carotid occlusion or artery-to-artery embolism from a thrombus on the intimal surface of the dissected artery. However, whether the mechanism of brain ischemia associated with aortic dissection is hemodynamic ischemia or thromboembolism remains unclear. Therefore, emergency surgical intervention for acute type A aortic dissection complicated by stroke remains controversial. However, when dissection involves the carotid arteries and compromises brain perfusion, flow can only be re-established within a short therapeutic window in the setting of acute stroke.
A)

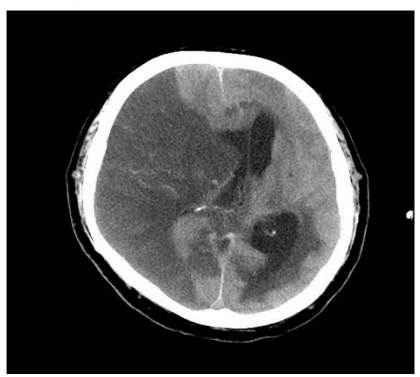

B)

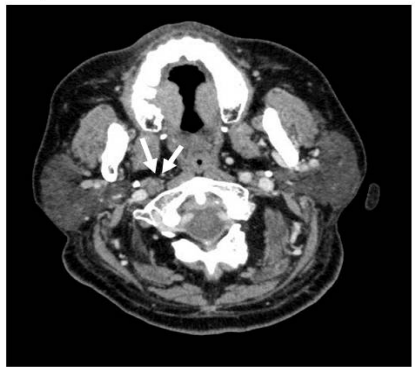

Figure 2: CT images.

A) Post-operative brain CT image shows area of extensive right hemispheric low-density.

B) Large free-floating thrombus in right internal carotid artery before operation (arrow).
Several investigators have reported favorable outcomes of the immediate surgical repair of acute type A dissection complicated by cerebral malperfusion [2,3]. However, several patients even in their series developed postoperative neurological deterioration and eventual cerebral death. The effects of cardiopulmonary bypass and reperfusion on the ischemic brain have not been elucidated. The optimal method of protecting the brain during aortic arch surgery for patients with cerebral malperfusion remains unclear. Estrera and associates reported $19 \%$ and $50 \%$ operative mortality and neurological recovery rates, respectively, among 16 patients after aortic repair under profound hypothermic arrest and retrograde cerebral perfusion [2]. Pocar and associates reported $0 \%$ mortality and an $80 \%$ neurological recovery rate for comatose patients who underwent surgery with ASCP [3]. Several factors, including the time to treatment, collateral circulation, extent of ischemia, and stroke subtype, might influence the response to reperfusion. Our patient already had a large free-floating thrombus in the right internal carotid artery before surgery. The natural history of free-floating thrombus in the carotid artery is unknown [4]. Freefloating thrombus is particularly challenging for physicians because most patients present with acute neurological symptoms and whether surgical or medical treatment would be more beneficial is unclear. We could not confirm how hypercoagulability related to cutaneous polyarteritis nodosa such as anticardiolipin antibody and lupus anticoagulant antibody was associated with the condition of this patient. However, thrombectomy should be performed before ASCP as described Parodi et al. [5]. We could not clarify why the ischemic stroke occurred, or whether the cerebral infarction or the blood flow decrease due to dissection or both were involved. However, we considered that a decrease in cerebral blood flow due to narrowing of the true lumen by a thrombosed pseudo lumen is one cause of ischemic stroke and that aortic dissection might cause acute ischemic stroke.

\section{References}

1. Fann JI, Sarris GE, Mitchell RS, ShumwayNE, Stinson EB, et al. (1990) Treatment of patients with aortic dissection presenting with peripheral vascular complications. Ann Surg 212: 705-713.

2. Estrera AL, Garami Z, Miller CC, Porat EE, Achouh PE, et al. (2006) Acute type A aortic dissection complicated by stroke: can immediate repair be performed safely? J Thorac Cardiovasc Surg 132: 1404-1408.

3. Pocar M, Passolunghi D, Moneta A, Mattioli R, Donatelli F (2006) Coma might not preclude emergency operation in acute aortic dissection. Ann Thorac Surg 81: $1348-1351$.

4. Bhatti AF, Leon LR Jr, Labropoulos N, Rubinas TL, Rodriguez H, et al. (2007) Free-floating thrombus of the carotid artery: literature review and case reports. J Vasc Surg 45: 199-205.

5. Parodi JC, Rubin BG, Azizzadeh A, Bartoli M, Sicard GA (2005) Endovascular treatment of an internal carotid artery thrombus using reversal of flow: a case report. J Vasc Surg 41: 146-150. 